\title{
Aproximaciones de la sociología de género hacia la violencia de la mujer en Lima Metropolitana
}

\section{RESUMEN}

En Lima Metropolitana las interacciones que se dan entre la mujer y el varón se producen y reproducen al interior de la familia, ahí se construyen las representaciones sociales y culturales sobre lo femenino y masculino. También en la familia se configura la sexualidad, los comportamientos violentos, las normas y los valores. Asimismo, advertimos cómo desde la aparición de la propiedad privada, el matrimonio monógamo, la familia patriarcal y relaciones de poder, genera la inequidad del varón en relación a la mujer quien se considera superior, y se extinguen las oportunidades de las mujeres para desarrollarse plenamente en la vida social. La Organización Mundial de la Salud y la Organización Panamericana de la Salud (1998) demuestran que el problema de la violencia hacia la mujer se inicia desde la niñez, continúa en la adolescencia, la etapa reproductiva hasta la senectud. Además, la OMS (2016) destaca que el Perú, ocupa el tercer lugar en el mundo por violencia sexual y feminicidio, después de Etiopía y Bangladesh.

Palabras clave: familia, representaciones sociales-culturales, femenino, masculino, sexualidad, familia patriarcal, relaciones de poder, violencia sexual y feminicidio.

\section{Approaches of Gender Sociology towards Violence of Women, in Metropolitan Lima}

\begin{abstract}
In Metropolitan Lima the interactions that take place between women and men are produced and reproduced within the family, where social and cultural representations of the feminine and masculine are constructed. Also in the family, sexuality, violent behavior, norms and values are configured. We also notice how from the emergence of private property, monogamous marriage, the patriarchal family and relations of power; generates the inequality of the male in relation to the woman who is considered superior, and the opportunities of women are extinguished to develop fully in the social life. The World Health Organization \& the Pan American Health Organization (1998) show that the problem of violence against women starts from: childhood, continues in adolescence, reproductive stage to old age. In addition, the World Health Organization (2016) points out that Peru ranks third in the world for sexual violence and femicide, after Ethiopia and Bangladesh.
\end{abstract}

KeYwords: family, social-cultural representations, feminine, masculine, sexuality, patriarchal family, power relations, sexual violence and feminicide. 


\section{Introducción}

L a complejidad del tema de la violencia hacia la mujer no solo debe ser vista desde el aspecto legal y operadores de justicia, sino también a partir del enfoque socio-cultural, de tal manera que pueda revelarse a qué se debe la violencia y el feminicidio ${ }^{1}$ hacia las mujeres.

En ese sentido, en la primera parte de este artículo ponemos énfasis en cómo se construye socialmente el comportamiento de mujeres y varones desde la jerarquización de los roles atribuidos a cada sexo; la reproducción de la violencia hacia la mujer se naturaliza desde la familia, donde se internaliza y percibe como un ser inferior, extendiéndose está perspectiva y comportamiento a otras instituciones sociales como: la escuela, los medios de comunicación social y grupos de pares. Asimismo, señalamos las expresiones y formas de violencia que existe hacia la mujer desde su niñez hasta su senectud, afectando de esta manera su desarrollo como ser humano.

\section{La violencia hacia la mujer se aprende en la familia}

Como sostiene Joan W. Scott (1996) el sexo es biológico ${ }^{2}$, coexiste en nosotros desde que nacemos. Sin embargo, el género es una construcción social y cultural sobre los roles determinados que debemos de cumplir como mujeres y varones. En consecuencia: «el género es una categoría social impuesta sobre un cuerpo sexuado». Es decir, se llega a ser mujer y varón a través de un proceso histórico. Ser varón o mujer son categorías que la sociedad nos asigna, son los roles que debemos cumplir a lo largo de toda nuestra vida. Ello es la base de esta perspectiva y condición de la mujer en la actualidad, tal como lo refiere la feminista Simone de Beauvoir ${ }^{3}$ : «No se nace mujer, se llega a serlo».

El proceso de socialización se da en las familias durante la infancia. Allí se aprende a comportarse

1 Es cuando se produce el asesinato de una mujer por razón de su sexo. Diccionario de la Real Academia de la Lengua.

2 Citado en: Género una categoría útil para el análisis histórico en www. fundacionhenrydunant.org/images/stories/biblioteca.

3 Activista feminista del siglo xx, que con su libro El segundo Sexo abrió campo a las teorías y estudios de género. como mujer y varón, ya que cada uno tiene características particulares designadas desde la propia familia, y más tarde desde los demás agentes socializadores como la escuela, los medios de comunicación, los amigos y las mismas instituciones sociales.

Este es un proceso que se inicia en la infancia, ahí cada persona va reconociendo, adquiriendo, fortaleciendo su identidad sexual, y poseyendo determinados significados para sí. "Durante la primera socialización, y a lo largo de la adquisición del lenguaje, el sujeto incorpora las actitudes y las definiciones de los otros. Los valores de su medio devienen en sus propios valores $[. .$.$] . En este estadio de desarrollo,$ las representaciones son incorporadas masivamente dado que el sujeto no puede interponer ninguna distancia entre un yo ya constituido y las definiciones e instrucciones que él o ella está recibiendo. Por lo tanto, estos contenidos son internalizados como la realidad en si misma. Este conjunto de representaciones provee a cada sujeto de marcos de referencia a través de los cuales otorgará significado a sus experiencias futuras»". Ello es reforzado con lo que sostiene San Segundo (2008) con la designación de cada sexo, en la mujer existe el mito de la sensibilidad, ternura, "comprensión para con las debilidades del varón $»^{5}$, en cambio en él se reconoce su poder seductor, afán conquistador que acompaña la violencia por ser varón, subyaciendo la idea de que la mujer tiene como cualidad su intuición femenina y más bien no le concierne el sentido lógico y racional que indiscutiblemente corresponde por excelencia al varón.

Los designios de cada sexo del «qué hacer» y «no hacer», comienzan desde el hogar (la familia) hasta los espacios abiertos. Es así que, la socialización en la familia autoritaria ${ }^{6}$, escuela y barrio, se aprenden básicamente conductas respecto a lo que significa ser femenino o masculino en un contexto social y cultural, en el cual la mujer aparece bajo relaciones de subordinación con respecto al varón.

En este contexto, predominan los padres que imponen las normas sin mediar explicaciones; la frase común es: "porque yo te lo digo» y el incumplimien-

4 Fuller, Norma, Masculinidades, cambios y permanencias. Varones de Cuzco, Iquitos y Lima. Fondo Editorial PUCP, Lima 2001,

5 Las comillas son nuestras: existen mecanismos sociales o representaciones que seńalan que el comportamiento de la mujer debe ser sensible a las debilidades o actitudes irracionales del varón.

6 Según la clasificación de tipos de Familia que hace la Socióloga Violeta Sara-Lafosse Valderrama. 
to de éstas suele acompañarse de amenazas o castigos, se caracteriza por tener un alto nivel de control, baja expresión de comunicación y afectividad hacia los hijos, las consecuencias son; que ellos se mantienen obedientes y sumisos cuando perciben un control externo de esta naturaleza, aunque al desaparecer éste presentan conductas agresivas.

Las mujeres se encuentran dependientes de la organización familiar. En esta prevalece un modelo autoritario que pone énfasis en la construcción de identidades como «sujetos subordinados», condicionando a sus miembros a un proyecto de vida personal, e imponiendo en la relación de pareja inequidades de género y centralidad en la hegemonía de las decisiones del varón 7 .

La violencia hacia los hijos es resultado de la relación de agresión y/o violencia en sus diferentes manifestaciones en el hogar de origen y de la pauta de comportamiento que impone el padre en la relación con la madre. Existe un estudio en España que concluye lo siguiente: «Vivir en contextos de violencia de género afecta al ajuste psicosocial de los/las menores y deteriora las competencias parentales de las víctimas. En consecuencia, la intervención de los/las profesionales del ámbito sociosanitario con las víctimas debería prestar mayor atención a las dificultades de los/las menores y a restablecer las competencias de crianza de las madres en aras de paliar las repercusiones de la violencia de género en sus hijos/as» ${ }^{8}$. Además, los autores mencionan que las situaciones de violencia en los que vive la madre determinan que las relaciones de afecto hacia los hijos/as se vean quebrantadas,

7 «La visión «androcéntrica» del mundo separa a mujeres y hombres y refuerza estereotipos de unas y otros según los roles de género que deben cumplir en las diversas esferas de la vida pública y privada. En: Sexismo y Androcentrismo puntogenero.inmujeres.gob.mx. Consultado el 8 de diciembre del 2018. La palabra androcentrismo hace referencia a la práctica, consciente o no, de otorgar al varón y a su punto de vista una posición central en el mundo, las sociedades, la cultura y la historia (...). Desde una perspectiva androcéntrica, los hombres constituyen el sujeto de referencia y las mujeres quedan invisibilizadas o excluidas. Es una visión referida también al lenguaje y a las instituciones sociales. Asimismo, es una perspectiva de todos los que han sido socializados en esta visión». Véase: Acevedo Huertas, Emilio José (2010). La transmisión del androcentrismo en los procesos de enseńanza-aprendizaje formales (II). Consultado en Revista digital para profesionales de la enseńanza 12.12. 2018. www. feandalucia.ccoo.es/docu/p5sd7196.pdf

8 Véase: Rosser Lumiñana Ana; Surià Martínez Raquel \& Mateo Pérez Miguel Ángel. Problemas de Conducta Infantil y Competencias Parentales en Madres en Contextos de Violencia de Género Gac. Sanit. 2018; 32 (1): 35-40. generándose mayor dificultades en los mismos, durante el aprendizaje (poca atención y concentración) y la disposición en romper las normas y producir conductas agresivas. Un hecho relevante que resaltan los autores refiriéndose a Mazza et al. ${ }^{9}$ es cómo los problemas socioeconómicos producen conflictos en la salud mental de las madres, generando situaciones de estrés en la familia e interferencias en la calidad de crianza de los hijos.

Con respecto a la educación que reciben los varones en la familia, Ramos (2006), sostiene que: «durante la nińez, ellos aprenden a reprimir sus emociones y no las expresan; muchas veces los hombres sienten cólera o rabia, cuando en realidad tienen miedo, tristeza, aburrimiento o desilusión; pero estas expresiones no pueden ser exteriorizadas por el varón porque son interpretadas como vulnerabilidad y debilidad de su autoridad y expuestos al ridículo; incentivándolos a incursionar en el ámbito público. Mientras que a las mujeres se les promueve lo contrario, exteriorizar sus emociones, asumir funciones domésticas, el cuidado y protección a los hijos principalmente, relejándola al espacio de lo privado y a la reproducción, aspecto poco valorado en el mundo social y económico» ${ }^{10}$. Por su parte, Kaufman (1989) destaca ${ }^{11}$ que: «el miedo a perder el control por parte de los varones en el ámbito familiar frente a las mujeres consideradas como seres con menor poder ${ }^{12}$ social y músculos débiles, no representa una amenaza física o psíquica para ellos». Conllevando a una perspectiva desigual y de inferioridad sistemática, en la cual se fundamenta las diferencias entre ambos sexos.

Es así que, las relaciones de subordinación de las mujeres se basa en un sistema cultural patriarcal ${ }^{13}$ que

9 Mazza JR, Pingault JB, Booij L, et al. Poverty and behavior problems during early childhood: the mediating role of maternal depression symptoms and parenting. International Journal of Behavioral Development. 2016:1-11.

10 Refiérase a Ramos Padilla, Miguel (2006). Masculinidades y violencia conyugal. Experiencia de vida de hombres de sectores populares de Lima y Cusco. Universidad Peruana Cayetano Heredia. Pag.16.

11 Kaufman, Michael (1989). Hombres. Placer, poder y cambio. Centro de Investigación para la Acción Femenina (CIPAF). Santo Domingo.

12 Max Weber. 1964, 2da. Edición, página 43. Poder: «significa la probabilidad de imponer la propia voluntad, dentro de una relación social, aun contra toda resistencia y cualquiera que sea el fundamento de esa probabilidad».

13 Deviene del patriarcado entendido como un estrato simbólico estructura inconsciente que conduce los afectos y distribuye valores entre los personajes del escenario social. En los conceptos básicos encontramos a una organización social primitiva en que la autoridad es ejercida por un varón jefe de cada familia, extendiéndose este poder 
predomina históricamente, ello surgió con el advenimiento de la agricultura; y exigiendo que el varón asumiera roles productivos y más bien, a ellas se les relegó a un rol no productivo, lo doméstico y privado; incrementándose el dominio social del varón y modificando la estructura familiar que se volvió patriarcal. Parafraseando lo dicho por Engels, "Con la aparición de la propiedad privada, del matrimonio monogámico y de la familia patriarcal, entraron en juego nuevas formas sociales, tanto en la sociedad en su conjunto, como en la organización familiar, que abolieron los derechos que tenían la mujer» (Reed, 1980). Este sistema que según San Segundo (2008) impedía a las mujeres instruirse, disponer libremente de sus bienes privados, trabajar y administrarlos sin la ausencia del marido.

En ese sentido, como lo define Sau: «la construcción social que otorga mayor poder a los varones y plantea una posición de subordinación a las mujeres, es la base de la violencia de género [...] una toma de poder histórico por parte de los hombres sobre las mujeres, cuyo agente ocasional fue de orden biológico, elevado este a categoría política y económica. Dicha asunción de poder pasa forzosamente por el sometimiento de las mujeres a la maternidad, la represión de la sexualidad y la apropiación de la fuerza de trabajo total del grupo dominado, del cual su primer pero no único producto son los hijos» ${ }^{14}$. Sin embargo las diferencias biológicas no justifican las desigualdades entre ambos sexos, como creadora de vida, la mujer tuvo la capacidad de organizar y dirigir las primeras formas de vida social:

«Durante un periodo en el cual los hombres se ocupaban exclusivamente de la caza y de la guerra, las mujeres desarrollaron la mayor parte de los instrumentos, de los conocimientos y las técnicas que estaban en la base del progreso social [...]». La gran variedad de artes que practicaban, se incluye la alfarería, el curtido, los tejidos, la construcción de vivienda, etc; fueron ellas las que desarrollaron los rudimentos de la botánica, la química y la medicina y otras ramas del conocimiento científico. (Ibídem, pág. 70).

a los parientes aun lejanos de un mimo linaje (RAE). Asimismo, es un sistema de creencias en un orden jerárquico que refuerza la superioridad masculina sobre la mujer y lo femenino, asumiendo control sobre los derechos individuales y sociales de los demás (Naciones Unidas).

14 Victoria Sau. (1989). Diccionario Ideológico Feminista.

\section{Expresiones de la violencia hacia las mujeres}

La base de todo este sistema se encuentra en la estructura patriarcal, Rita Segato (2003) realiza un análisis la reproducción del género como estructura de relaciones entre posiciones marcadas por un diferencial jerárquico de otras clasificaciones como lo étnico o de clase, expresando lo siguiente:

El mero desplazamiento de la mujer hacia una posición no destinada a ella en la jerarquía del modelo tradicional pone en entredicho la posición del hombre en esa estructura, ya que el estatus es siempre un valor en un sistema de relaciones. Más aún, en relaciones marcadas por el estatus, como el género, el polo jerárquico se constituye y realiza justamente a expensas de la subordinación del otro (ibídem, pág. 31).

La autora plantea la tesis estructural de la violencia de género, teniendo en cuenta que la posición del varón en sociedades jerárquicas tradicionales no permite la movilidad vertical de las mujeres a mejores posiciones por ser tales; por tanto, se establecen relaciones sociales de oposición y desigualdad entre ellos.

Ello explica las relaciones de género en la actualidad, la subordinación es parte del sistema, por eso el énfasis que, la violencia va en pirámide, de lo más simple (formas sutiles/invisibles) control, exclusividad, aislamiento, anulación, chantaje emocional, desprecios, humillaciones y amenazas; hasta las más complicadas (formas visibles/complejas) como gritos, insultos, violación, abusos, agresión física y homicidios. En tal sentido, el hombre produce estrategias de dominación que harían posible la subordinación de la mujer, cuando ella no se está en una relación de inferioridad.

En el Perú, según la Endes: «El 41.5\% de las mujeres agredidas físicamente y sexualmente refirieron que el inicio de la violencia por parte del actual/último esposo o compañero fue luego de transcurrido uno a dos años de unión o convivencia» ${ }^{15}$.

El Ministerio de la Mujer y Poblaciones Vulnerables (MIMP), destacó que la causa de la violencia del varón contra la mujer, se debe básicamente a relaciones de poder de inequidad, en donde el varón se asume superior a la mujer (Plan Nacional

15 Instituto nacional de Estadística e Informática. Encuesta Demográfica y de Salud Familiar-ENDES, 2011. Lima, Perú. 
Contra la Violencia Hacia la Mujer para el período 2009-2015); en ese sentido, existe en este una actitud de posesión hacia la mujer. Por ejemplo, la violencia psicológica obedece a alguna forma de control del esposo o compañero hacia la mujer, que se expresa en el afán de saber dónde van las mujeres y sentir celos hacia ellas. Así como palabras humillantes, el impedimento de que visite a sus familiares, la amenaza de abandono, privarla del aporte económico; afecto de sus hijos o de acusarlas de infidelidad.

Para el MIMP y el Centro Emergencia Mujer (CEM), las denuncias por violencia física hacia la mujer han sido aproximadamente 50 a mediados de noviembre del 2017. Y las consecuencias de la misma a nivel de agresiones físicas, traumas psicológicos y situaciones de pobreza o extrema pobreza, son atendidas por profesionales especializados en Psicología, Abogacía, Trabajo Social y especialistas en promoción social, que asesoran y brindan terapias a las mujeres para superar los efectos de la violencia en su desarrollo personal y familiar.

Sin embargo, son sólo paliativos, no resuelven el problema debido a que como sabemos se continúan presentando casos de violencia hacia la mujer por parte del varón. En el distrito de San Juan de Lurigancho, de enero a marzo del 2015, se atendió un total de mil casos de violencia familiar y sexual, de 309 casos; 50\% fueron reportados por violencia psicológica.

\section{Violencia hacia la mujer a través de su ciclo vital}

Como podemos notar las expresiones de violencia son múltiples y ello se visibiliza en Lima, ya que concentra la tercera parte de la población peruana, estimando que en el ańo 2017, el 65,4\% de las mujeres de 15 a 49 años de edad, fueron víctimas de violencia ejercida alguna vez por el esposo o compañero; registrándose una disminución de 2,8 puntos porcentuales al compararlo con el año $2016^{16}$, estas cifras crecen cuando se trata de la violencia psicológico ${ }^{17}$

16 Instituto Nacional de Estadística e Informática. Encuesta Demográfica de Salud Familiar (ENDES), Junio del 2018, Lima Perú.

17 Las formas más frecuentes de violencia psicológica ejercida alguna vez por el esposo o compañero fueron: ejercer algún control $(57,1 \%)$ insistir en saber a dónde va $(40,4 \%)$, manifestación de celos $(37,9 \%)$ e impide que visite o la visiten sus amistades (18,7\%) INEI-ENDES, 2017. Lima, Perú. en un $57,1 \%$ en Lima Metropolitana. En general, el (MIMP) registró en el mismo año en Lima 15,424 casos de violencia exclusivamente en mujeres, llegando a formas más extrema de violencia como el feminicidio de la mujer, con 149 víctimas en el $2018^{18}$.

Por lo que vemos la violencia, abuso y feminicidio hacia la mujer es una situación que se presenta a lo largo del ciclo vital como lo presentamos y resumimos en el cuadro de la siguiente página ${ }^{19}$.

Como podemos observar tenemos diferentes tipos y expresiones de violencia, agresiones y hasta muerte de las mujeres, durante el ciclo vital de la mujer, incluso se presenta a partir de su estado prenatal; es decir, desde su concepción hasta su último día de existencia.

Con respeto a estos tipos de expresiones de violencia, el Campo de Salud de la Mujer de la OMS y OPS (1998) mencionan que: «la violencia tiene un efecto profundo sobre la mujer. Empieza antes del nacimiento, en algunos países, con abortos selectivos según el sexo. $\mathrm{O}$ al nacer, cuando los padres desesperados por tener un hijo varón pueden matar a sus bebés del sexo femenino. Y sigue afectando a la mujer a lo largo de su vida. Todos los ańos, millones de nińas son sometidas a la mutilación de sus genitales. Las niñas tienen mayor probabilidad que sus hermanos de ser violadas o agredidas sexualmente por miembros de su familia, por personas en posiciones de poder o confianza, o por personas ajenas. En algunos países, cuando una mujer soltera o adolescente es violada, puede ser obligada a contraer matrimonio con su agresor, o ser encarcelada por haber cometido un acto "delictivo». La mujer que queda embarazada antes del matrimonio puede ser golpeada, condenada al ostracismo o asesinada por sus familiares, aunque el embarazo sea producto de una violación. Después del matrimonio, el riesgo mayor de violencia para la mujer sigue habitando en su propio hogar, donde su esposo y, a veces la familia política, puede agredirla, violarla o matarla. Cuando la mujer queda embaraza-

18 Referencia proporcionada por los Centros de Emergencia Mujer (CEM) a nivel nacional, del Programa Nacional Contra la Violencia Familiar y Sexual del Ministerio de la Mujer y Poblaciones Vulnerables.

19 Cuadro elaborado a partir del artículo: «Género y Violencia: un enfoque desde la salud pública» de la Organización Panamericana de la Salud, 1999. Y del informe de:» Violencia contra la mujer. Un tema de salud prioritario» de la OMS, 1998. 


\section{VIOLENCIA DE GÉNERO EN EL CICLO VITAL}

\section{ETAPA PRENATAL}

- Golpizas durante el embarazo, tiene efectos en el resultado del nacimiento del ser humano.

- Embarazo no deseado.

- Preferencia por el hijo varón, rechaza a las niñas desde su nacimiento.

\section{ETAPA DE LA INFANCIA}

- Abuso físico y emocional.

- $\quad$ El acceso diferencial a la alimentación entre la nińa y el nińo.

- La prioridad en el tratamiento médico y en la educación para varones

- $\quad$ sobre las niñas.

\section{ETAPA DE LA NIÑEZ}

- Abuso sexual por parte de miembros de la familia y extraños.

- El acceso diferencial a la alimentación entre la niña y el nińo.

- La prostitución infantil.

- La carga de las tareas domésticas solo a niñas.

\section{ETAPA DE LA ADOLESCENCIA}

- La violencia de los compańeros del sexo opuestos.

- La violación sexual por un miembro de familia, por un extraño o pareja.

- $\quad$ La prostitución forzada.

- $\quad$ El tráfico de mujeres.

- $\quad$ Sexo forzado por razones económicas (niñas estudiantes que tienen relaciones sexuales con adultos a cambio de favores).

- Incesto.

- El acosamiento sexual.

- La prostitución y pornografía forzada.

\section{ETAPA REPRODUCTIVA}

- $\quad$ El abuso de parte de su pareja.

- La violación conyugal.

- $\quad$ El homicidio conyugal.

- El maltrato psicológico.

- $\quad$ Los aborto por maltrato.

- abuso sexual en el sitio de trabajo.

- El acosamiento sexual.

- La prostitución y pornografía forzada.

- Tráfico de mujeres.

- $\quad$ Abuso de mujeres discapacitadas.

- $\quad$ Abuso y homicidio.

\section{ETAPA DE LA VEJEZ}

- En el abuso y maltrato contra las ancianas y ancianos.

- La violación.

- $\quad$ El abandono.

- La violencia psicológica.

- «El suicidio forzado u homicidio de viudas por razones económicas» ${ }^{1}$ da, envejece o padece discapacidad mental o física, es más vulnerable al ataque» ${ }^{20}$.

Pudiendo denotar en cada etapa de su vida afrontan tipos de violencia no solo psicológica y física, sino que estas se presentan según la edad y cabe referir que cada una de ellas, traerá consecuencias directas e indirectas para el desarrollo de sus vidas y su comunidad.

Esta expresión de violencia hacia la mujer, ha posicionado al Perú en el tercer país del mundo con mayor incidencia en delitos por violaciones sexuales y feminicidios después de Etiopía y Bangladesh ${ }^{21}$. Asimismo, según el MIMP: «Perú es el segundo país con mayor violencia sexual en la cual se registra un total de 19675 casos de violencia contra la mujer entre el periodo 2014-2015. Y en Junio del 2018, la región que registra mayor índice de violencia sexual es Lima con 749 casos, en segundo lugar Junín con 156 casos y en tercer lugar, Cusco con 144 casos». ${ }^{22}$

\section{Conclusiones}

La presencia de las familias autoritarias en la sociedad peruana, tienden a reproducir los mismos comportamientos y concepciones; sobre lo que es ser varón y mujer, esbozando la probabilidad de que se generen interacciones marcadas por agresiones, violencia $y$ feminicidio.

La educación que reciben tanto los varones como las mujeres en las familias e instituciones sociales, produce y refuerzan la construcción de roles diferenciados en la interacción social, en tanto unos son autoritarios y otras sumisas.

La violencia y el feminicidio hacia la mujer responde a una estructura patriarcal, que tiene una concepción ideológica y práctica social, que refuerza la posición de superioridad y la relación de poder del varón con respecto a la mujer.

El control y el poder que el varón expresa en las relaciones de pareja hacia la mujer, es resultado de

20 Organización Mundial de la Salud \& Organización Panamericana de la Salud (1998). Violencia Contra la Mujer. 08.12. 2018 https:// www.whoint/gender/violence/violencia infopack1.pdf. Pág. 4-5.

21 Zavala, J. OMS (2016). 08.03.2018 https://diariocorreo.pe/edicion/ lima/peru-tercer-lugar-en-el-mundo-por-violaciones-sexuales-y-feminicidios-807011/

22 Ministerio de la Mujer y Poblaciones Vulnerables 26.04.2017 https://larepublica.pe/sociedad/1035525-peru-es-el-segundo-paiscon-mayor-violencia-sexual 
la ausencia de un apego seguro ${ }^{23}$ desde los primeros años de la infancia.

Existen espacios institucionalizados en la sociedad peruana, en los cuales se construye, reproduce y fomenta la «violencia» hacia la mujer; en los que se genera la identidad sexual masculina y afectos, que no permiten la valoración de la mujer como ser humano, en igualdad de oportunidades en la relación de pareja con el varón.

\section{Bibliografía}

Acevedo Huertas, Emilio José (2010). La transmisión del androcentrismo en los procesos de enseńanza-aprendizaje formales (II). Consultado en Revista digital para profesionales de la enseñanza 12.12.2018 www.feandalucia.ccoo.es/docu/p5sd7196.pdf

Blossiers Mazzini, Carolina (2007). Interacción entre el personal de Salud y las Gestantes Adolescentes durante el Control Prenatal desde los Significados de la Sexualidad, el Embarazo y la Atención Prenatal en Hospital Nacional. Tesis para optar el Grado de Magíster en Género, Sexualidad y Salud Reproductiva. Universidad Peruana Cayetano Heredia. Lima.

Bourdieu, Pierre (2000). La dominación masculina, en www.nomasviolenciacontramujeres.cl/wp-content/ uploads/2015/09/

Buitrón Aranda, Gabriela (2003). Identidad y maternidad. Estereotipos de género, maternidad adolescente y barreras ante la planificación familiar. Asociación Peruana de Salud Mental para la Mujer-Sección de Salud Mental de la Mujer de la Asociación Mundial de Psiquiatría. Lima.

Careaga GP (1996). Las relaciones entre los géneros en la salud reproductiva. México DF. Comité Promotor por una Maternidad sin Riesgos en México.

CMP Flora Tristán (2005). La violencia contra la mujer: Feminicidio en el Perú. Lima: Flora Tristán.

Consi, Jorge (1994). Una mirada abarcativa sobre el problema de la violencia familiar. En Corsi, Jorge (Compilador). Violencia familiar. Una mirada inter-

23 La persona siente confianza en situaciones adversas, tiene sentimientos de pertenencia y buena autoestima con quien mantiene vínculos, respuestas afectivas, ayuda y comprensión. Josu Gago. Teoría del apego. El vínculo. 06.12.18. www.avntf-evntf.com/wp-content/ uploads/2016/06/Teoría-del... disciplinaria sobre un gran problema social. Argentina: Editorial Paidós, pp. 15-83.

Dutton, Donald \& Golant, Susan (1999). El golpeador. Un perfil psicológico. Buenos Aires: Editorial Paidós. Psicología, Psiquiatría y Psicoterapia 170.

Figueroa \& Liendro (1995). La presencia del varón en la salud reproductiva. In: Hardy E, Osis MJ, Crespo ER (Eds.). Ciências Sociais e Medicina. Atualidades e Perspectivas LatinoAmericanas. Campinas, Centro de Pesquisas das Doenças Materno-Infantis de Campinas, Foucault, Michel (2000). Historia de la sexualidad 1: La voluntad del saber. México: Siglo Veintiuno Editores.

Fuller, Norma (1997). Identidades masculinas. Varones de clase media en el Perú. Lima: Pontificia Universidad Católica del Perú. Fondo editorial.

Fuller, Norma (2001). Masculinidades, cambios y permanencias. Varones de Cuzco, Iquitos y Lima. Lima: Pontificia Universidad Católica del Perú. Fondo editorial.

Gustin, Matos, Godillo, Chávez y Talavera (1978). Recién nacido de madre adolescente. Population CouncilNew York. Lima.

Instituto Nacional de Estadística e Informática (2011/2017). Informe Endes. Lima. Perú.

Jacobson, Niel \& Gottman, John (2001). Hombres que agreden a sus mujeres. Cómo poner fin a las relaciones abusivas. Barcelona, Buenos Aires: Ed. Paidós.

Kaufman, Michael (1989). Hombres. Placer, poder y cambio. Santo Domingo: Centro de Investigación para la Acción Femenina (CIPAF).

Martínez García, María Ángeles y Camarero, Luis Alfonso (2015). La reproducción de la violencia de género: una lectura desde las áreas rurales. En: Revista de Estudios sobre Despoblación y Desarrollo Rural / Journal of Depopulation and Rural Development Studies. Universidad Nacional de Educación a Distancia. DOI: 10.4422/ager.2015.12 15.12.2018 https://reivindicaciones.files.wordpress.com/2015/07/la-reproduccic3b3n-de-la-violencia-de-gc3a9nero-una-lectura-desde-las-c3a1 reas-rurales.pdf

Matamala, María Isabel \& Salazar, Gloria (1994). Afectividad, género y maternidad en las adolescentes. En 1er Congreso de Salud Nacional Mujer y Salud Mental. SERAM, Chile, pp. 157-160.

Mazza JR, Pingault JB, Booij L, et al. Poverty and behavior problems during early childhood: the mediating role of maternal depression symptoms and parenting. 
International Journal of Behavioral Development. 2016:1-11.

Ministerio de la Mujer y Poblaciones Vulnerables 26.04.2017https://larepublica.pe/sociedad/1035525peru-es-el-segundo-pais-con-mayor-violencia-sexual

MOP. Perfil psicológico del hombre violento. 20.09.2018 https://www.mop.cl/GIS/Documents/ PREVENCION_DE_LA_VIOLENCIA_UNA_ TAREA_DE_TODOS.pdf

Moreno, Hortensia (2001). Relaciones Sexuales En: Sexualidad y Derechos Ciudadanos. Universidad Nacional Mayor de San Marcos- Flora Tristán, Centro de la Mujer Peruana.

OMS (1998). «Género y Violencia: un enfoque desde la salud pública» de la Organización Panamericana de la Salud. Y del informe de:» Violencia contra la mujer. Un tema de salud prioritario».

Perú es el segundo país con mayor violencia sexual. La República. 26.12.2018 https://larepublica.pe/ sociedad/1035525-peru-es-el-segundo-pais-con-mayor-violencia-sexual

Ramos Padilla, Miguel (2006). Masculinidades y violencia conyugal: Experiencia de vida de hombres de sectores populares de Lima y Cusco. Lima: Universidad Peruana Cayetano Heredia.

ReEd, Evelyn (1980). Sexo contra sexo o clase contra clase. Espańa: Editorial Fontamara.
Rosser Lumiñana, Ana; Surià Martínez, Raquel \& Mateo Pérez, Miguel Ángel (2018). Problemas de conducta infantil y competencias parentales en madres en contextos de violencia de género. Gac. Sanit.; 32 (1): 35-40.

San Segundo, Manuel Teresa (2008). Violencia de género. Una visión multidisciplinar. Madrid: Editorial Ramón Areces.

Sara-Lafosse, Violeta (1978). La familia y la mujer en contextos sociales diferentes. Lima: Departamento de Ciencias Sociales. Pontificia Universidad Católica del Perú.

SAU, Victoria (1989). Diccionario ideológico feminista. Barcelona, 2da. Edición, pp. 237-238.

Segato, Rita (2003). Las estructuras elementales de la violencia. Argentina: $1 .^{\circ}$ ed. Bernal: Universidad Nacional de Quilmes.

URP (2017). Violencia contra la mujer en el distrito de Santiago de Surco-Lima. Lima: Universidad Ricardo Palma.

Viveros, Mara (2002). De quebradores y cumplidores: Sobre hombres, masculinidades y relaciones de género en Colombia. Bogotá: Universidad Nacional de Colombia.

Weber, Max (1964). Economía y Sociedad. México: Fondo de Cultura Económica.

Zavala, J. OMS (2016). 08.03.2018 https://diariocorreo. pe/edicion/lima/peru-tercer-lugar-en-el-mundo-porviolaciones-sexuales-y-feminicidios-807011/ 